\title{
Factores de distribución de las comunidades del bosque húmedo de montaña. Volcán Cofre de Perote, México
}

\author{
Distribution factors in fragmented cloud forest communities in Cofre de Perote volcano, Mexico
}

\author{
Jennifer K Walte-Vega a, Arturo García-Romero a*, Lilia L Manzo-Delgado a , José López-García a \\ *Autor de correspondencia: ${ }^{a}$ Universidad Nacional Autónoma de México, Instituto de Geografía, \\ Departamento de Geografía Física, Circuito Exterior s/n, Colonia Ciudad Universitaria, México, Ciudad de México, C.P. 04510, \\ tel.: (52-55) 56224335 ext. 45461, agromero@igg.unam.mx
}

\section{SUMMARY}

\begin{abstract}
The mountain cloud forest, also known as cloud forest, is one of the terrestrial ecosystems with a distribution restricted to mountainous elevations with high precipitation and persistent fog. Due to physical, ecological and cultural changes in the landscape, the communities that exist today represent a minimum fraction of the ecosystem. The objective of this investigation was to identify the factors related to the geomorphological diversity and to changes related to cultural processes that act as key drivers of the distribution of cloud forest communities in one of central Mexico's highest peaks. Floristic composition of plant communities was determined through 21 samplings, including ecotone areas, disturbed sites and even coffee plantations located within the forest. These communities were mapped using GIS techniques, and their distribution was assessed considering various factors pertaining to relief, land use and fragmentation. Five major communities were identified: 1) Carpinus tropicalis + Quercus delgadoana forest, 2) Carpinus tropicalis + Prunus serotina forest, 3) Alchornea latifolia + Liquidambar macrophylla ecotone, 4) Cupressus lusitanica + Alnus jorullensis ecotone and 5) coffee plantations grown under the forest canopy. Relevant environmental factors were grouped into two axes: one comprising the spatial structure (fragmentation) and the other involving the morphological characteristics of relief. Altitude and the energy of relief are the environmental factors most significantly related to changes in the specific richness of the communities studied.
\end{abstract}

Key words: Cofre de Perote volcano, mountain cloud forest, cloud forest, forest fragmentation, geomorphological diversity.

\section{RESUMEN}

El bosque húmedo de montaña o bosque de niebla es uno de los ecosistemas terrestres de distribución restringida a elevaciones montañosas con altas precipitaciones y niebla persistente. Debido a cambios físicos, ecológicos y culturales, las comunidades conservadas representan una proporción mínima del ecosistema. El objetivo del estudio fue reconocer los factores relativos a la diversidad geomorfológica y a los cambios provocados por procesos culturales, que controlan la distribución de las comunidades del bosque húmedo de montaña, en una de las montañas más altas del Centro de México. Para conocer la composición florística de las comunidades se realizaron 21 muestreos, incluyendo zonas de ecotono, lugares con evidencia de perturbación e incluso, cafetales que presumiblemente se encuentran dentro del bosque. Mediante técnicas de SIG se generó el mapa de las comunidades, y se analizó su distribución con respecto a diversos factores referentes al relieve, el uso del suelo y la fragmentación. Se identificaron cinco comunidades: 1) bosque de Carpinus tropicalis y Quercus delgadoana, 2) bosque de Carpinus tropicalis y Prunus serotina, 3) ecotono con Alchornea latifolia y Liquidambar macrophylla, 4) ecotono con Cupressus lusitanica y Alnus jorullensis, y 5) cafetales bajo el dosel del bosque. Los factores que fueron relevantes se agruparon en dos ejes, uno referente a la estructura espacial (fragmentación), y otro a las características morfológicas del relieve. La altitud y la energía del relieve son los factores que se relacionaron de forma más significativa con cambios en la riqueza específica de las comunidades.

Palabras clave: volcán Cofre de Perote, bosque húmedo de montaña, bosque de niebla, fragmentación forestal, diversidad geomorfológica.

\section{INTRODUCCIÓN}

La composición de una comunidad vegetal está controlada por factores físicos y biológicos, incluyendo procesos y patrones tanto de origen natural como antrópico que intervienen dentro del sistema ambiental e interactúan de manera no lineal (Farina 1998). De manera particular, la adaptación de las plantas a diferentes altitudes se considera como una respuesta a las condiciones climáticas (Hovenden y Brodribb 2000), siendo de gran interés analizar la distribución de las comunidades vegetales en relación a la heterogeneidad del relieve y la fragmentación, considerados ambos como fiables controladores de la distribución de ciertos elementos climáticos como la radiación, 
la temperatura, la precipitación y la humedad (Turner et al. 2001). Sin embargo, es de conocimiento general que la influencia de dichos factores cambia con la escala de estudio; por ejemplo, a escala regional la orografía es uno de los principales factores físicos que inciden en la distribución y características del clima, y de las comunidades (Csorba 2010), en tanto que otros factores como los relativos a las características de la litología o el suelo no son particularmente evidentes (Rzedowski 2006).

Pero a escala de paisaje (escala intermedia entre las escalas regional y local; 1:25.000 a 1:75.000) las relaciones relieve-vegetación son más específicas, siendo la heterogeneidad topográfica y litológica la que mejor se relaciona con los cambios en la distribución de los organismos vegetales (Huston 2005, Williams-Linera 2007). Así, en general, se acepta que tipos de vegetación parecidos ocurren en ambientes geomorfológicos que son similares (Turner et al. 2001, García-Aguirre et al. 2010). Esto se debe a que las geoformas de distinto origen se asocian a cambios en las condiciones ambientales, por ejemplo, en la composición y estabilidad del sustrato geológico, en las propiedades físicas y químicas del suelo, en los niveles de evaporación del agua, la disponibilidad de humedad y la estabilidad de laderas y erosión, todos los cuales influyen en el establecimiento y desarrollo de la vegetación (Corenblit et al. 2008, Newman et al. 2014).

Por otra parte, a mesoescala los factores culturales también inciden en las características de la vegetación, y existe un amplio consenso en aceptar que en la mayoría de los casos esta influencia ha sido negativa al intervenir el hombre como factor de disturbio (Turner et al. 2001). Además, la deforestación y la pérdida gradual de la continuidad y la conectividad de los fragmentos remanentes pueden tener severas consecuencias en la biodiversidad y alteraciones en la estructura y en la funcionalidad de las comunidades (Hilty et al. 2006, Echeverría et al. 2007). Los daños se incrementan al considerar que el contacto de los fragmentos con la matriz circundante transforma las condiciones ambientales (i.e. temperatura y humedad) y favorece la influencia de procesos externos (Farina 1998), dando como resultado el aumento de la heterogeneidad de condiciones, organismos y desarrollo entre los distintos fragmentos (Collinge 2009).

Un ejemplo de la situación descrita se tiene en los bosques húmedos de montaña, también conocidos como bosques de niebla o bosques nubosos del centro de México, incluyendo el caso de los fragmentos forestales de la vertiente occidental del volcán Cofre de Perote. El área se caracteriza por su cercanía al litoral del Golfo de México y por un gradiente altitudinal muy pronunciado entre las costas y las cumbres del volcán (100 km y > 4.000 m de desnivel), situación que incide en las complejas condiciones impuestas por la circulación general de la atmósfera predominante (sistemas tropicales en verano y de latitudes medias en invierno), permitiendo la existencia de diversos tipos de vegetación: bosque tropical, bosque templado y pastizal de alta montaña (García-García y Montañez 1991, Rzedowski 2006). Entre ellos, el bosque húmedo de montaña ha sido reconocido como un ecotono entre la vegetación de la región biogeográfica Neártica y la Neotropical, lo que le confiere una gran diversidad y riqueza biológica (Toledo 2009). Esta diversidad se incrementa no solo por su distribución en distintas áreas geográficas, sino también porque la fragmentación histórica del ecosistema ha favorecido una diversidad muy alta de epifitas, arbustos y herbáceas, además de numerosos endemismos (Cayuela et al. 2006, Toledo 2009), siendo difícil encontrar consistencias florísticas entre fragmentos de distintas regiones, e incluso entre fragmentos cercanos (Cayuela et al. 2006).

El interés por su conservación radica en ser un ecosistema de distribución restringida a elevaciones montañosas (menos del $1 \%$ del territorio nacional), al tiempo que intensamente afectado por procesos de cambio de uso del suelo (Rzedowski 2006). La tasa de cambio de los bosques húmedos de montaña a uso agrícola es muy alta en el país, tanto para los bosques primarios $(1,94 \%)$ como para los secundarios (6,28\%); mientras que su recuperación a partir de usos de suelo agrícolas es mínima $(0,05 \%)$ y la degradación de bosques primarios es alta (16,5 \%). En consecuencia, el $50 \%$ de la superficie original del bosque húmedo de montaña ha sido remplazada, siendo considerado por el gobierno mexicano como un ecosistema que requiere de atención especial (Toledo 2009).

Si bien el relieve, el uso del suelo y la fragmentación han sido destacados por su influencia en la ecología de las comunidades vegetales (Jules y Shahani 2003, GarcíaAguirre et al. 2010), aún no se han llegado a establecer las variables específicas que ejercen una influencia dominante en la composición vegetal del bosque húmedo de montaña en México; debido a ello y considerando la importancia ecológica de este tipo de vegetación, se eligió un área representativa ubicada en región central del país, para analizar la influencia de dichos factores. El objetivo es reconocer a nivel de paisaje el efecto que tienen los factores geomorfológicos, el uso del suelo y la fragmentación, sobre la distribución de las comunidades del bosque húmedo de montaña de la vertiente oriental del volcán Cofre de Perote (centro-occidente de México). El estudio se desarrolló bajo la hipótesis de que a escala del paisaje, la variabilidad del relieve controla la distribución de los patrones de uso de suelo y fragmentación, luego provocando heterogeneidad en la composición y riqueza de las comunidades.

\section{MÉTODOS}

Sitio de estudio. Se estudió un cuadrante $\left(672 \mathrm{~km}^{2}\right)$ de la vertiente oriental del volcán Cofre de Perote, ubicado a $\sim 200 \mathrm{~km}$ al Este de la Ciudad de México. El desnivel local de más de más de 3.000 m (860 - 4.200 m s.n.m) permite la transición de los climas cálido-húmedo a los templadohúmedo, y la formación de diversos tipos de vegetación, incluyendo selva mediana caducifolia, bosques de pino, 
encino, pino-encino, abeto, y pastizal de alta montaña. El bosque húmedo de montaña se distribuye entre $1.200 \mathrm{y}$ $2.100 \mathrm{~m}$ s.n.m. coincidiendo con el sector de la vertiente donde las masas de aire húmedo provenientes del Golfo de México generan las mayores precipitaciones orográficas $(1.500 \mathrm{~mm}$ año-1) y una persistente neblina a nivel del suelo (Williams-Linera 2007).

Un aspecto peculiar de esta vertiente es el notable control geomorfológico del patrón paisajístico. La organización geomorfológica se caracteriza por el predominio de geoformas modeladas sobre estructuras volcánicas asociadas a las distintas fases de formación del volcán Cofre de Perote (Plioceno tardío al Holoceno) y del campo volcánico monogenético Xalapa (Cuaternario), en tanto que solo el $5 \%$ está conformado por calizas del Mesozóico. Dos colapsos masivos originaron extensos depósitos heterolíticos de gran impacto en la geomorfología de la vertiente, uno de ellos asociado a una avalancha del volcán Cofre de Perote, y otro a un abanico de derrubios del Complejo Volcánico Las Cumbres.

La inspección preliminar del patrón paisajístico mostró extensas masas forestales ( 40 \% del área en estudio) bien conectadas entre sí (figura 1), así como sectores donde la expansión agrícola y pecuaria se traduce en una extensa matriz de cultivos y pastizales con fragmentos remanentes de bosque. Una actividad que ha tenido fuerte impacto en el paisaje es el cultivo de café ( $40-60 \%$ de la superficie del sector central del estado de Veracruz), el cual se encuentra bajo diversos tipos de sistemas productivos, desde los rústicos en los que no se altera el estrato arbóreo, hasta los monocultivos de sol (Rzedowski 2006).

Muestreo de vegetación. Para representar la diversidad de comunidades posibles en los bosques húmedos de montaña primarios, secundarios y con manejo, se realizaron 21 muestreos en sitios de ecotono, lugares con evidencia de perturbación e incluso, cafetales que se encuentran dentro del bosque. La existencia de ecotonos se explica por las variaciones climáticas que afectan a las vertientes del volcán. De esta forma, 14 sitios de muestreo se ubicaron en la franja de clima templado húmedo C(f) (García 1973), donde la topografía de valles incididos y barrancos favorece una mayor humedad. Otros siete sitios de muestreo se ubicaron a menor altitud, en zonas de transición hacia la franja de clima semicálido húmedo (A)C(fm) (García 1973). Se consideró como criterio para la selección de los

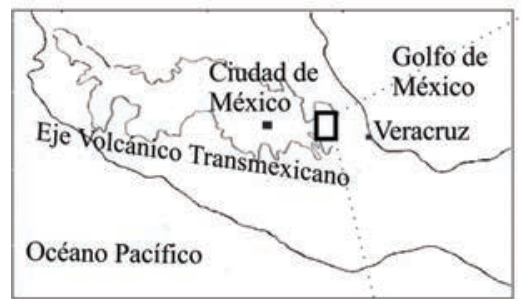

Leyenda

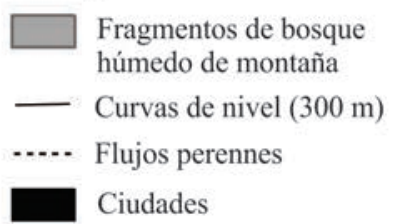

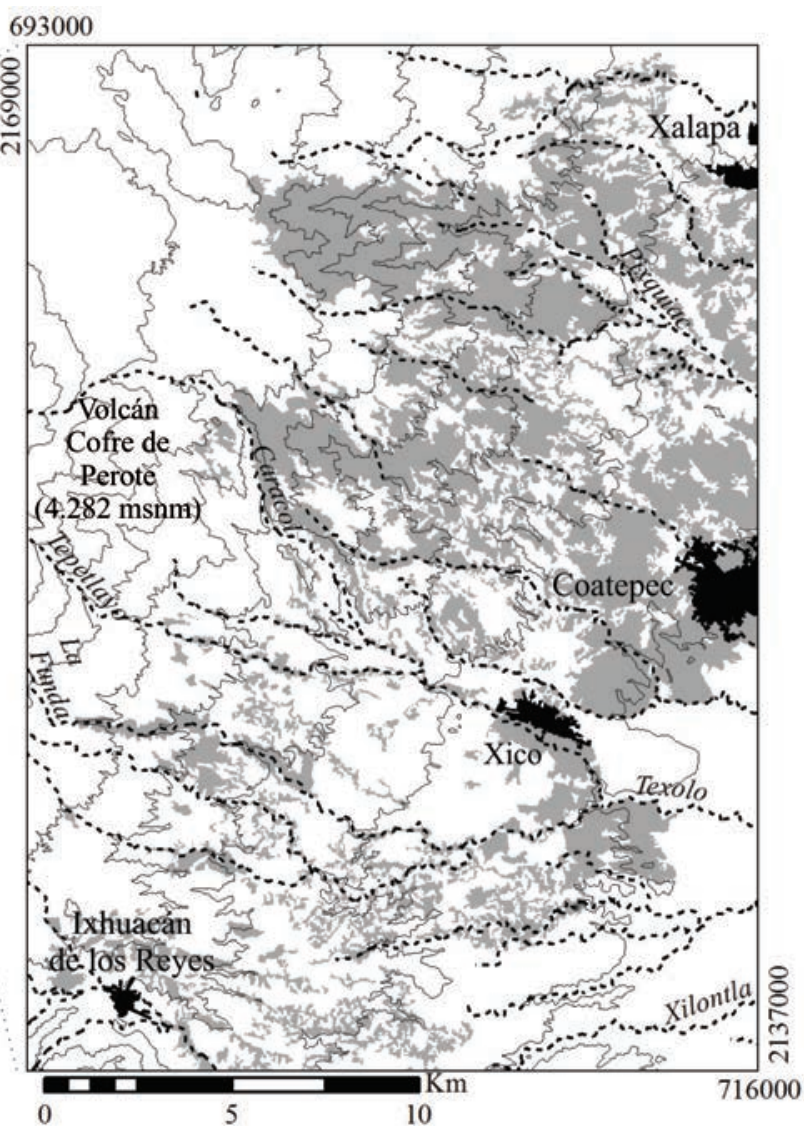

Figura 1. Distribución de fragmentos del bosque húmedo de montaña en la vertiente oriental del volcán Cofre de Perote, México. Distribution of mountain cloud forest on the eastern slope of the Cofre de Perote volcano, Mexico. 
sitios la presencia de elementos característicos del bosque, como es el caso de los helechos arborescentes y diversas especies de epifitas que son indicadores de humedad, así como de ciertas especies de coníferas de afinidad neártica y de algunas latifoliadas de afinidad tropical. Si bien las especies herbáceas forman parte de la diversidad de los sitios, en este caso la investigación consideró solo árboles y arbustos, al ser estos los elementos más estables y permanentes de la comunidad. En cada sitio se realizó un transecto de $100 \mathrm{~m}$ de largo por $2 \mathrm{~m}$ de ancho para el registro de los individuos del estrato arbóreo; y al centro del transecto un cuadrante de $25 \mathrm{~m}^{2}$ para los individuos del estrato arbustivo. Las muestras fueron identificadas en el Herbario de la Facultad de Ciencias de la Universidad Nacional Autónoma de México.

Clasificación de comunidades. Para determinar las comunidades del bosque húmedo de montaña se calculó una matriz de distancias entre sitios de muestreo a partir de las abundancias de todas las especies en los distintos sitios. Se utilizó el índice de disimilitud de Bray-Curtis, el cual ha demostrado ser uno de los más adecuados para realizar ordenaciones altamente robustas, tanto para datos continuos como agrupados (Faith et al. 1987). Además, se realizó un cluster jerárquico a la matriz de distancias para agrupar los sitios de muestreo de acuerdo a la composición florística. El análisis crea subgrupos exclusivos a su rama superior, asegurando de este modo que dichos subgrupos sean homogéneos al interior y heterogéneos al exterior de la rama (Ludwig y Reynolds 1988).

Análisis cartográfico de factores de distribución. Para determinar los factores que explican la distribución de las comunidades del bosque húmedo de montaña a nivel del paisaje, se elaboró una serie cartográfica con cinco mapas que se refieren a la organización geomorfológica de la vertiente estudiada y dos mapas que se refieren al uso del suelo y la fragmentación forestal.

El mapa geomorfológico se interpretó a partir de ortofotos digitales de 1995 (2 m de resolución) y de los mapas topográfico (curvas de nivel a cada $20 \mathrm{~m}$ ) y geológico (SGM 2007) a escala 1:50.000. Se consideraron tres aspectos del análisis morfogenético (Tapia-Varela y López Blanco 2002): a) tipo de geoforma, b) geología (unidades homogéneas en términos de su origen, litología y edad), y c) morfometría. Para la caracterización morfométrica de las unidades del relieve se elaboraron cinco mapas: altimetría (metros), pendiente del terreno (\%), energía del relieve (máxima diferencia en altura, en metros), densidad de drenaje $\left(\mathrm{km} \mathrm{km}^{-2}\right)$ e insolación (WH m-2) (Tapia-Varela y López-Blanco 2002).

El mapa de fragmentos del bosque húmedo de montaña del 2012 se obtuvo mediante la interpretación de las ortofotos digitales, y el mapa resultante fue actualizado sobre compuestos de color de las imágenes Landsat TM del 2012. Las imágenes fueron corregidas geométricamente y georreferenciadas a mapas topográficos (1:50.000) mediante el método "Tie-Points", y se utilizó el índice SIG$\mathrm{MA} \leq 2$ para verificar la precisión. Se aplicó un método de interpretación visual, el cual consiste en técnicas directas, asociativas y deductivas para delimitar los fragmentos de bosque, y la verificación de los fragmentos resultantes se realizó mediante trabajo de campo, literatura e indicadores como pendiente del terreno, altimetría y exposición de laderas.

Se incluyeron todos los fragmentos forestales con resolución de superficie $>1$ ha $(100 \times 100 \mathrm{~m})$ y conectados por corredores de ancho $>10 \mathrm{~m}$ y longitud $<50 \mathrm{~m}$. Finalmente, se utilizó el programa FRAGSTATS 4.0 (McGarigal et al. 2012) para analizar las métricas espaciales de tamaño, forma, conectividad y contraste de los fragmentos de bosque. Además, para evaluar el impacto asociado al uso del suelo se elaboró un mapa de densidad de caminos (longitud de vías por $\mathrm{km}^{2}$ ), considerado como un indicador del disturbio.

Selección de factores de distribución. Se consideraron 17 factores, cinco de los cuales se relacionaron con la organización geomorfológica de la vertiente estudiada (altitud, pendiente, insolación, energía del relieve y densidad del drenaje), y 12 con el uso del suelo y a las características espaciales de la fragmentación: densidad de caminos, ÁREA (superficie del fragmento), PERIM (perímetro), PARA (tasa perímetro-área), SHAPE (índice de forma), FRAC (dimensión fractal perímetro-área), CORE (área de zonas núcleo en un fragmento, donde: área > $50 \mathrm{~m}$ desde cualquier borde de fragmento), NCORE (número de zonas núcleo en un fragmento), CAI (porcentaje del área de un fragmento ocupada por las zonas núcleo), PROX (relación ENN/ÁREA del fragmento y de los fragmentos vecinos del mismo tipo), ENN (distancia euclidiana del vecino más cercano), y ECON (porcentaje de fragmentos de la misma clase que se encuentran separados por una distancia menor que el umbral establecido en 1.000 m) (figura 2). Se aplicó un análisis de correlación de Pearson y un PCA (análisis de componentes principales), con la finalidad de seleccionar a las variables que explican la mayor cantidad de varianza sin estar correlacionadas. A estas variables se les aplicó un MANOVA no paramétrico permutativo utilizando la matriz de distancias como variable de respuesta, de las cuales se seleccionaron aquellas con efectos significativos como factores de distribución de las comunidades del bosque húmedo de montaña.

Modelación de la distribución espacial del bosque húmedo de montaña. Se realizó un escalamiento multidimensional no métrico (NMDS por sus siglas en inglés) con la matriz de distancias para modelar las diferencias entre sitios de acuerdo a las abundancias de todas las especies (NMDS). El análisis crea un espacio multidimensional en el cual cada dimensión representa la abundancia de una especie en cada sitio. Así, se crea una configuración de 


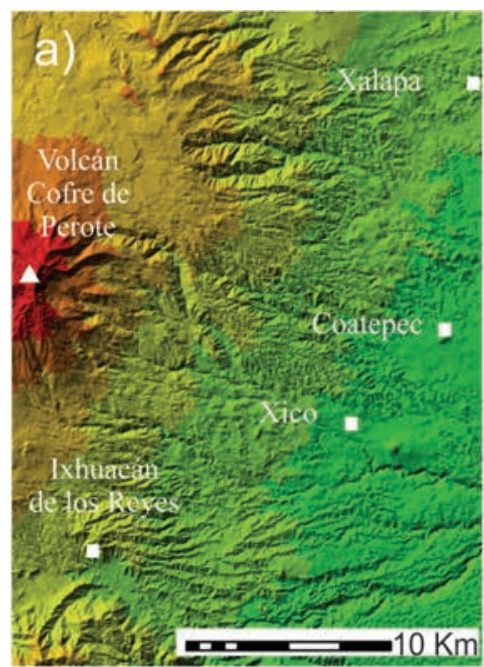

Altimetría (msnm)
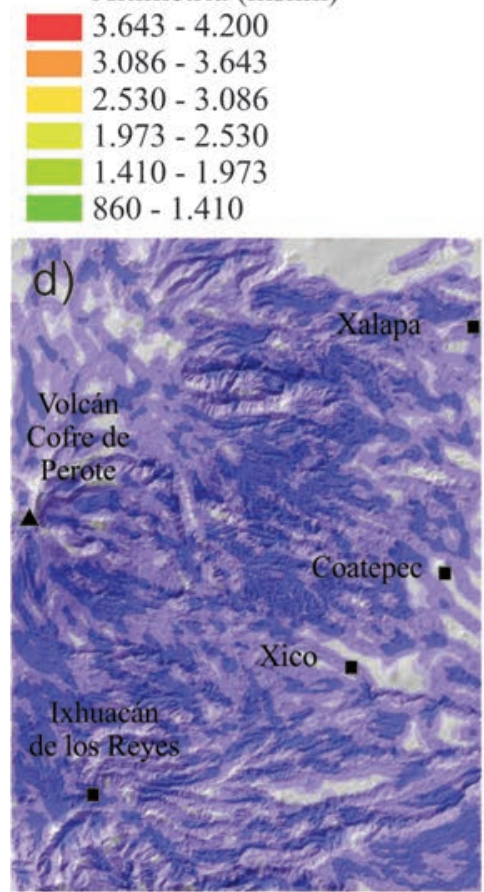

Densidad del drenaje $\left(\mathrm{Km} / \mathrm{Km}^{2}\right)$

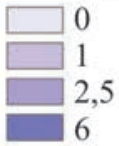

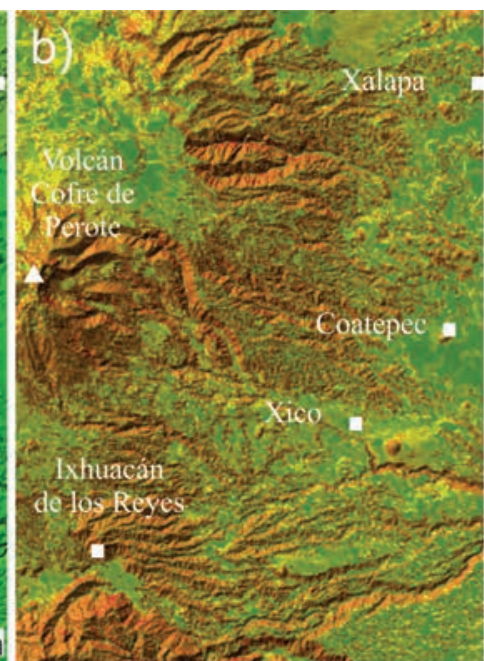

Pendientes (grados)

0 - 3 Muy bajas

3 - 6 Bajas

6 - 15 Moderadas

15 - 45 Altas

45 - 90 Muy Altas

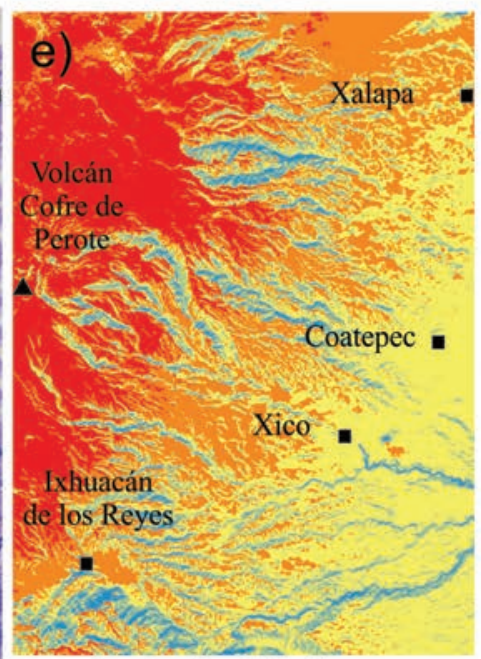

Insolación $\left(\mathrm{W} / \mathrm{m}^{2}\right)$

$187.485-650.000$

$650.000-750.000$

$750.000-840.000$

$840.000-910.000$

$910.000-1.106 .747$

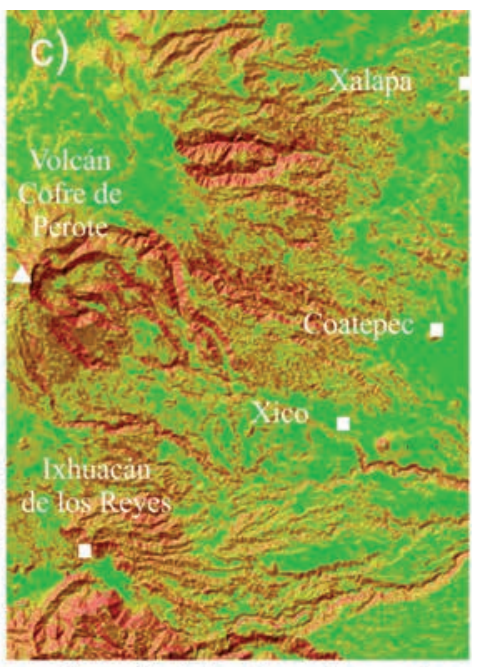

Energía del relieve (metros)

0 - 20

$20-40$

$40-80$

$80-140$

$140-240$

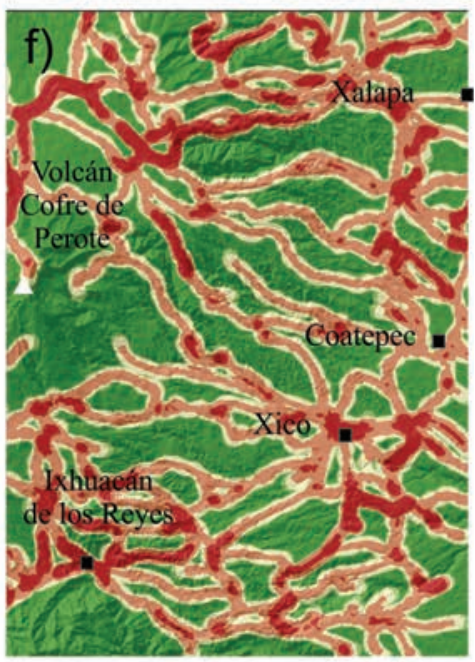

Densidad de caminos $\left(\mathrm{Km} / \mathrm{Km}^{2}\right)$

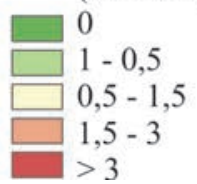

Figura 2. Selección de 6 mapas de factores de distribución del bosque húmedo de montaña, referentes al relieve (A - E) y el uso del suelo (F).

Maps of 6 main distribution factors of cloud mountain forest, concerning the relief (A - E) and land use (F). 
puntos distribuidos en este espacio de acuerdo a la cantidad de individuos de cada especie, se calcula la distancia Euclidiana entre puntos y se obtiene otra matriz de distancias (d'). Después se ordenaron los puntos de la matriz d' de manera que se asemeje al orden de la matriz d, y las diferencias fueron medidas como estrés con la fórmula de Kruskal (1964). Este es un proceso iterativo en el que con cada iteración se busca reducir el estrés y se acepta la ordinación con menor estrés, la cual se normaliza y representa en un espacio bidimensional en el que la cercanía de los puntos indica similitud en composición (Faith et al. 1987, Ludwig y Reynolds 1988). Los factores de distribución (altitud y energía del relieve) fueron ajustados al modelo NMDS mediante superficies de tendencias (thin plate splines), que consiste en la formación de curvas suaves que reflejan la relación de los puntos en la ordenación y las variables que se ajustan (Wood 2003). Además, se realizó un árbol de clasificación con los factores de distribución y los tipos de comunidades, del que se obtuvieron umbrales de distribución por altitud y energía del relieve para cada tipo de comunidad de bosque húmedo de montaña, con el cual se realizó un mapa de distribución de comunidades.

\section{RESULTADOS}

Clasificación de comunidades del bosque húmedo de montaña. El análisis PCA permitió determinar la existencia en la vertiente estudiada de cinco comunidades, de las cuales dos fueron del bosque húmedo de montaña, otras dos fueron ecotonos con los pisos bioclimáticos que delimitan el sector bajo y alto de la vertiente y una tercera fue un agrosistema dentro del bosque: 1) bosque de Carpinus tropicalis (Donn. Sm.) Lundell y Quercus delgadoana S. Valencia-Avalos, Nixon et L.M. Kelly; 2) bosque de Carpinus tropicalis y Prunus serotina Ehrh.; 3) ecotono con Alchornea latifolia Sw. y Liquidambar macrophylla Oerst.; 4) ecotono con Cupressus lusitanica Mill. y Alnus jorullensis Kunth.; y 5) cafetales bajo dosel del bosque.

El bosque de Carpinus tropicalis y Quercus delgadoana se distribuyó sobre $1.300 \mathrm{~m}$ de altitud. Incluyó una mezcla de elementos tropicales y templados, aunque con predominio de éstos últimos. En el estrato arbóreo dominaron los individuos de Liquidambar macrophylla, $C$. tropicalis, Q. delgadoana y Prunus serotina, de afinidad templada, y Trichila sp. P. Browne y Rhamnus sp. L., de afinidad tropical, mientras que en el arbustivo dominaron los individuos del género tropical Deppea sp. Schltdl. et Cham. Presentó la mayor riqueza de helechos y epifitas, además de que en todos los sitios muestreados se presentaron helechos arborescentes.

El bosque de Carpinus tropicalis y Prunus serotina estuvo ubicado en laderas más bajas que el grupo anterior $(<1.300 \mathrm{~m}$ de altitud). El dominio de los elementos templados sobre los tropicales se suavizó, por ejemplo, en el estrato arbustivo dominó Moussonia deppeana (Schltdl. et Cham.) Hanst., de afinidad tropical, y Roldana sp. La Llave, de afinidad templada; mientras que en el arbóreo dominaron Ardisia liebmannii Oerst., de afinidad tropical, $C$. carolineana Thomas Walter, P. serotina y varias especies de Quercus de afinidad templada. Se caracterizó además, por una gran cantidad de epifitas, principalmente Bromelia sp. Adans. y Tillandsia sp., y por una alta riqueza de helechos, aunque se tuvieron helechos arborescentes en una menor proporción de sitios.

El ecotono de Alchornea latifolia y Liquidambar macrophylla se encontró cercano a los ambientes propios de las selvas. Se caracterizó por una mezcla de elementos tropicales, como A. latifolia, y de distribución más amplia como Trema micrantha (L.) Blume y Platanus mexicana Moric., además de algunos individuos aislados de L. macrophylla. Sin embargo, el bosque fue poco diverso y se presentó altamente perturbado, siendo notable la ausencia del estrato arbustivo. Por su parte, el ecotono de Cupressus lusitanica y Alnus jorullensis fue el único que presentó individuos de Pinus spp. L., Abies religiosa (Kunth) Schltdl. et Cham, C. lusitanica y Eupatorium sp. L. Los individuos del género Cestrum L. dominaron tanto en el estrato arbóreo como en el arbustivo y se presentaron algunos individuos del bosque húmedo de montaña como Liquidámbar sp. y una gran abundancia de helechos y epifitas.

Los cafetales bajo el dosel del bosque fueron poco diversos y poseyeron una mayor dominancia de elementos tropicales, como es el caso de Musa sp. L., Inga schiedeana Steud. y Alchornea latifolia, aunque en ocasiones solo presentaron individuos de Trema micrantha. Se observó una alta perturbación debido a las actividades humanas, primordialmente asociada a los asentamientos humanos, los cultivos de plátano dentro de los mismos cafetales y las zonas cubiertas con bambú. Aunque es evidente el dominio del café, también se encontraron individuos de Ceanothus depressus Benth. Algunos de estos sitios se encontraron en zonas en reposo, por lo que presentaron individuos de café sin evidencias de haber sido podados o aprovechados.

Las comunidades más diversas se encontraron en los bosques de Carpinus tropicalis y Quercus delgadoana, en los cuales se obtuvo un índice de diversidad de Simpson de 0,877 y de Shannon de 2,488. A ellos les siguieron los bosques de Carpinus tropicalis y Prunus serotina, el ecotono con Cupressus lusitanica y Alnus jorullensis, el ecotono con Alchornea latifolia y Liquidambar macrophylla, y finalmente los cafetales (cuadro 1).

Modelo de distribución de las comunidades del bosque húmedo de montaña. El PCA, utilizando cinco factores relativos a la organización geomorfológica de la vertiente y 12 factores relativos al uso del suelo y a las características espaciales de la fragmentación, explicó el total de la varianza en 14 componentes y el $57 \%$ en los dos primeros 
Cuadro 1. Promedio de los índices de diversidad por comunidad.

Average of diversity indices by community.

\begin{tabular}{lcccccc}
\hline Clase & $\begin{array}{c}\text { Número } \\
\text { de sitios }\end{array}$ & $\begin{array}{c}\text { Número de } \\
\text { individuos }\end{array}$ & $\begin{array}{c}\text { Número familias } \\
\text { de Riqueza }\end{array}$ & Simpson & Shannon \\
\hline Bosques de Carpinus tropicalis y Quercus delgadoana & 4 & 122 & 13 & 20,50 & 0,877 & 24,888 \\
Bosques de Carpinus tropicalis y Prunus serotina & 6 & 70 & 9 & 13,67 & 0,799 & 20,268 \\
Ecotono de Alchornea latifolia y Liquidambar macrophylla & 3 & 28 & 7 & 8,67 & 0,756 & 17,488 \\
Ecotono de Cupressus lusitanica y Alnus jorullensis & 3 & 62 & 3 & 11,00 & 0,736 & 18,077 \\
Cafetales & 5 & 51 & 6 & 8,40 & 0,576 & 1,3100 \\
\hline
\end{tabular}

componentes. En el primer componente se encontraron los factores asociados a la fragmentación, mientras que en el segundo, los relacionados con el relieve. Del componente fragmentación los factores que obtuvieron los valores más altos fueron el área, el perímetro, el índice SHAPE, el área y el número de zonas núcleo, el índice de conectividad y la proximidad. Del componente relieve los factores con valores más altos fueron la altitud, la energía del relieve y la pendiente (cuadro 2).

Sin embargo, se observó una correlación alta entre casi todos los factores de la fragmentación y el área del fragmento, así como entre el índice de proximidad y el de conectividad, y entre la pendiente y la energía del relieve. Por ello, para descartar aquellos factores sin efectos significativos sobre la riqueza y, por lo tanto, que no tienen efectos dominantes sobre la composición, se seleccionaron solo cuatro factores: altitud, energía del relieve, área del fragmento e índice de conectividad; a los cuales se aplicó un análisis MANOVA no paramétrico permutativo, donde dichos factores fueron considerados como variables independientes y la riqueza como variable de respuesta. El resultado demostró que la altitud $(\mathrm{F}=2,3245 ; P=0,002)$ y la energía del relieve ( $\mathrm{F}=1,6940 ; P=0,018$ ) fueron las variables ambientales con efectos significativos sobre la distribución de la riqueza de especies en el bosque húmedo de montaña (cuadro 3).
Cuadro 2. Valores de los factores en el PCA.

Values of factors in the PCA.

\begin{tabular}{lcc}
\hline \multicolumn{1}{c}{ Factor } & PC1 & PC2 \\
\hline Altitud & $-0,0598$ & 0,3194 \\
Densidad de caminos & 0,0789 & $-0,2405$ \\
Densidad del drenaje & 0,0159 & $-0,0754$ \\
Energía del relieve & $-0,1366$ & 0,4410 \\
Pendiente & $-0,0480$ & 0,4576 \\
Insolación & 0,0517 & $-0,2966$ \\
ÁREA & 0,3581 & 0,0952 \\
PERIM & 0,3631 & 0,0870 \\
PARA & $-0,2804$ & $-0,0184$ \\
SHAPE & 0,3668 & 0,0676 \\
FRAC & 0,3005 & 0,1049 \\
CORE & 0,3559 & 0,0979 \\
NCORE & 0,3674 & 0,0726 \\
CAI & 0,3104 & 0,0004 \\
PROX & $-0,0414$ & $-0,4085$ \\
ENN & $-0,1607$ & 0,1749 \\
ECON & 0,1306 & $-0,3124$ \\
\hline
\end{tabular}

Cuadro 3. Resultados del análisis MANOVA no paramétrico permutativo. Results of the non-parametric MANOVA permutational analysis.

\begin{tabular}{lccccc}
\hline \multicolumn{1}{c}{ Fuente de variación } & Grados de libertad & Suma de cuadrados & $\mathrm{F}$ & $\mathrm{R}^{2}$ & P \\
\hline Área & 1 & 0,3990 & 1,0547 & 0,04707 & 0,336 \\
ECON & 1 & 0,4318 & 1,1413 & 0,05094 & 0,257 \\
Altitud & 1 & 0,9596 & 2,5362 & 0,11320 & $0,003 * *$ \\
Energía del relieve & 1 & 0,6330 & 1,6730 & 0,07467 & $0,018 *$ \\
Residuo & 16 & 6,0535 & & 0,71411 & \\
Total & 20 & 8,4769 & & 1,00000 \\
\hline
\end{tabular}

** $=P<0,01 \mathrm{y} *=P<0,05$ indican efectos significativos sobre la riqueza de especies. 
En el análisis de escalamiento multidimensional no métrico (NMDS), el eje horizontal (NMDS1) dividió a las comunidades muestreadas en dos sectores. Un sector de afinidad tropical que estuvo determinado por la presencia de individuos de Alchornea latifolia, Trema micrantha, Inga schiedeana y otros pertenecientes a la familia Theaceae; y otro sector de afinidad templada, donde fueron determinantes los individuos de Alnus jorullensis, Eupatorium sp. y Cestrum spp. (figura 3).
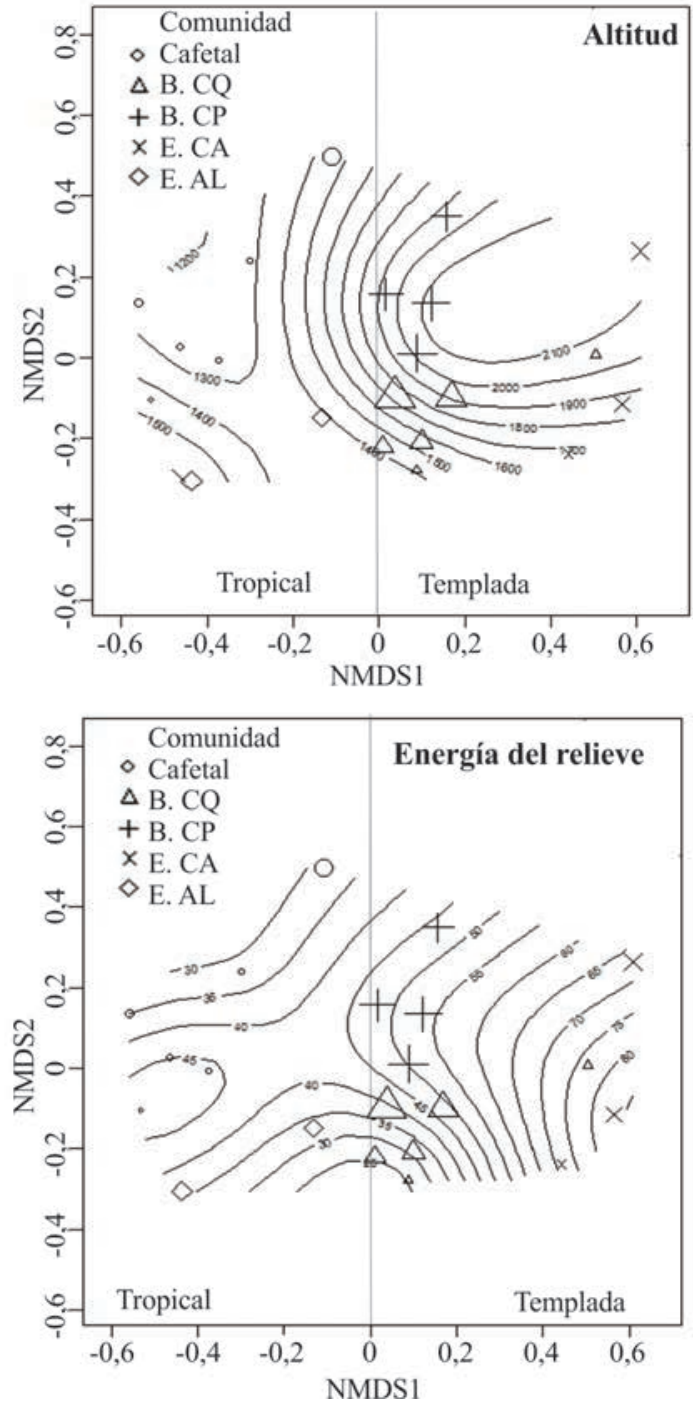

Figura 3. Modelos NMDS, con superficie de tendencias por altitud y energía del relieve. $\mathrm{B}$. CQ = bosque de Carpinus tropicalis y Quercus delgadoana; B. CP = bosque de Carpinus tropicalis y Prunus serotina; E. CA = ecotono de Cupressus lusitanica y Alnus jorullensis; y E. AL = ecotono de Alchornea latifolia y Liquidambar macrophylla.

NMDS model, with surface of trends by altitude and energy of relief. B. CQ = Carpinus tropicalis + Quercus delgadoana forest; B. $\mathrm{CP}=$ Carpinus tropicalis + Prunus serotina forest; E. CA $=$ Cupressus lusitanica + Alnus jorullensis ecotone; and E. AL = Alchornea latifolia + Liquidambar macrophylla ecotone.
El ajuste de la altitud y la energía del relieve al modelo NMDS permitió apreciar que en el sector con afinidad tropical, las diferencias entre grupos estuvieron dadas por la altitud, ya que mientras los cafetales se concentraron en zonas bajas (altitud $<1,400 \mathrm{~m}$ ), el ecotono de $\mathrm{Al}$ chornea latifolia y Liquidambar macrophylla se distribuyó a mayor altitud, aunque ambos grupos compartieron valores similares de energía del relieve (20 a $50 \mathrm{~m}$ ). En el sector de afinidad templada el grupo mejor diferenciado es el ecotono de Cupressus lusitanica y Alnus jorullensis, que se encontró a una altitud de 1.700 a $2.100 \mathrm{~m}$ y con energía del relieve mayor a $70 \mathrm{~m}$, en tanto que el bosque de Carpinus tropicalis y Quercus delgadoana se encontró en el mismo intervalo altitudinal pero con una energía del relieve menor de $60 \mathrm{~m}$, y el bosque de C. tropicalis y Prunus serotina se encontró en un intervalo más amplio de altitud (1.400 a $2.000 \mathrm{~m}$ ), aunque en zonas con menor energía del relieve $(<50 \mathrm{~m})$. Es de interés notar que las comunidades con la mayor riqueza vegetal: bosques de C. tropicalis y Q. delgadoana, y de C. tropicalis y P. serotina, se encontraron a altitudes entre 1.400 y $2.100 \mathrm{~m}$, mientras que en los extremos altitudinales del área de estudio se ubicaron las comunidades de menor riqueza. Así, en el caso de las zonas bajas, la relación altitud-riqueza fue contraria a la que se podría esperar; sin embargo, este resultado se puede explicar por un mayor impacto por parte de las actividades humanas dentro de estas áreas forestales, como es el caso del cultivo de café, plátano y bambú.

Reglas de distribución. El árbol de clasificación mostró que las comunidades del bosque húmedo de montaña se ajustaron a ciertas reglas de distribución que se establecieron de acuerdo a los intervalos de altitud y energía del relieve (figura 4). Este árbol tiene cuatro ramas en lugar de las cinco comunidades identificadas, pues existe una alta confusión entre los cafetales y el ecotono de Alchornea latifolia y Liquidambar macrophylla, los cuales se ubicaron a menos de $1.388 \mathrm{~m}$ de altitud. El ecotono de Cupressus lusitanica y Alnus jorullensis se ubicó a altitudes superiores a $1.388 \mathrm{~m}$ y donde la energía del relieve fue superior a los $66 \mathrm{~m}$. Esta fue la única comunidad que no posee ningún error de clasificación debido a la alta energía del relieve donde se distribuyó, característica que no comparte con las otras comunidades. Los bosques de Carpinus tropicalis y Q. delgadoana se encontraron por encima de $1.885 \mathrm{~m}$ de altitud y donde la energía del relieve no superó los $66 \mathrm{~m}$, lo cual puede resultar extraño dado que su composición presentó una mayor proporción de especies tropicales; sin embargo, puede ser un indicador de un mayor grado de conservación dada la inaccesibilidad de los sitios. Esta categoría tuvo un bajo porcentaje de confusión con los bosques de C. tropicalis y Prunus serotina, los cuales fueron muy parecidos en composición, aunque se distribuyen a altitudes inferiores a $1.885 \mathrm{~m}$ y con energía del relieve menor de $66 \mathrm{~m}$. 


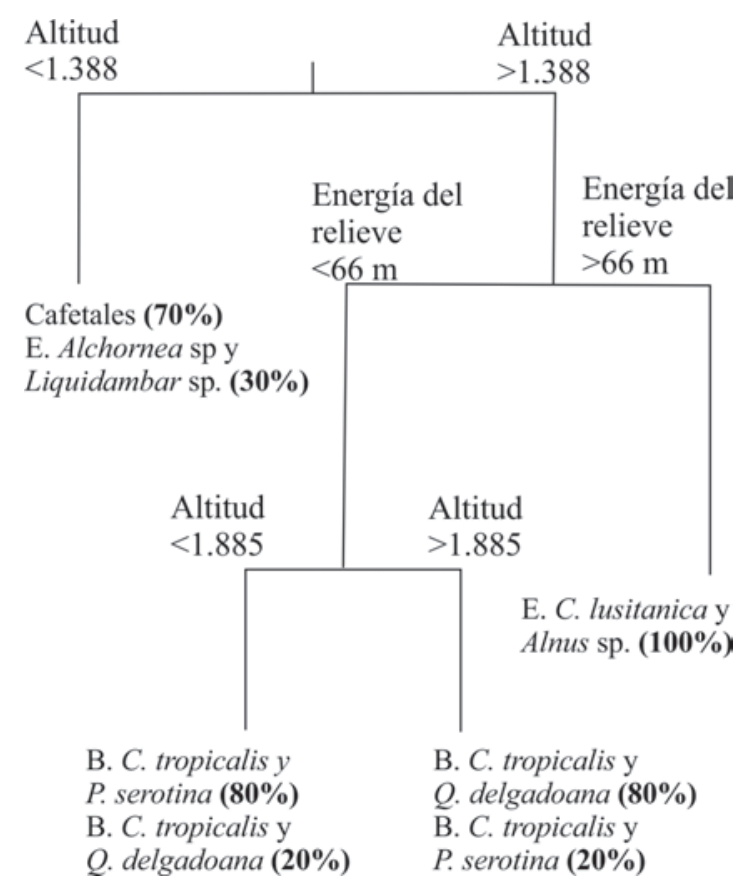

Figura 4. Árbol de distribución de las comunidades del bosque húmedo de montaña, según factores de distribución.

Distribution tree of mountain cloud forest communities, according to environmental factors.

\section{DISCUSIÓN}

El estudio de la composición florística permite identificar para el área de estudio cinco comunidades del bosque húmedo de montaña. Los bosques de Carpinus tropicalis y Quercus delgadoana y los de C. tropicalis y Prunus serotina tienen características que los asemejan a los bosques caducifolios, es decir, que poseen árboles caducifolios de afinidad boreal que dominan sobre los perennifolios, como es el caso de C. tropicalis y Liquidambar macrophylla (Challenger y Soberón 2008). Estos bosques se diferencian entre sí por la dominancia de elementos templados y mayor diversidad y equitatividad en los bosques de C. tropicalis y $Q$. delgadoana, mientras que en los de C. tropicalis y $P$. serotina dominan los elementos tropicales, al tiempo que existe una menor diversidad y equitatividad. Además, se identifican dos ecotonos y un agrosistema (cafetales), los cuales no presentan la estructura típica del bosque, sin embargo, comparten el mismo piso altitudinal, además de algunas similitudes altamente significativas como la presencia de helechos arborescentes aislados, alta cantidad de epifitas y especies como Liquidambar macrophylla, Quercus spp., y Carpinus caroliniana (Rzedowski 2006).

Los factores relevantes para explicar las diferencias entre comunidades se pueden agrupar en dos ejes, uno conformado por la estructura espacial (fragmentación) derivada de la expansión del uso del suelo, y otro por las ca- racterísticas morfológicas del relieve. Sin embargo, ambos ejes tienen efectos comprobados a escalas diferentes, por ejemplo, los efectos del relieve son más evidentes a escalas amplias (por ejemplo escala de región) e intermedias (escala de paisaje) (Huston 2005), mientras que los efectos de la fragmentación lo son a escalas locales, como en el caso de las diferencias de composición entre fragmentos (Collinge 2009).

Una de las manifestaciones más relevantes de la influencia del relieve en las características de las comunidades a escala regional son las distintas comunidades del bosque húmedo de montaña, las cuales se distribuyen en tres pisos altitudinales que responden al control orográfico del gradiente bioclimático de la vertiente estudiada (Rzedowski 2006, Csorba 2010). Es interesante notar que el umbral altitudinal de $1.388 \mathrm{~m}$ genera dos grupos de comunidades determinados fundamentalmente por cambios de temperatura. Mientras que los bosques de Carpinus tropicalis y Quercus delgadoana y el ecotono de Cupressus lusitanica y Alnus jorullensis (2.300 - $1.885 \mathrm{~m}$ de altitud), así como el bosque de $C$. tropicalis y Prunus serotina (1.885 - 1.388 m s.n.m.), coinciden con la distribución del clima C(f) y una temperatura media anual de 12 a $18^{\circ} \mathrm{C}$; por otra parte, los cafetales y el ecotono de Alchornea latifolia y Liquidambar macrophylla $(1.388-1.200 \mathrm{~m}$ de altitud) se distribuyen en una zona de transición bioclimática hacia el tipo $(\mathrm{A}) \mathrm{C}(\mathrm{fm})$, con temperatura media anual mayor de $18{ }^{\circ} \mathrm{C}$ y temperatura del mes más frío menor a $18^{\circ} \mathrm{C}$ (García 1973). Estos resultados confirman otros estudios que reportan a la altitud como un factor dominante en la composición del bosque húmedo de montaña (Challenger y Soberón 2008), por ejemplo, a partir de la altitud se han definido gradientes altitudinales y comunidades discretas (Alcántara et al. 2002).

En cambio, la influencia de la energía del relieve en la vegetación es particularmente evidente a escala de paisaje, y se relaciona con la heterogeneidad topográfica entre barrancos y lomas que ocurren dentro de los pisos bioclimáticos definidos por la altitud (Turner et al. 2001, Huston 2005). Una de las manifestaciones más relevantes se refiere a los cambios en humedad provocados por la energía del relieve, la cual crea zonas tipo "refugio" de muy baja insolación y con una alta concentración de cauces, como ocurre en el ecotono de Cupressus lusitanica y Alnus jorullensis. Esta idea se sustenta con otros resultados obtenidos en comunidades de bosques templados al sur de la Ciudad de México, donde la influencia de la humedad sobre la distribución de las comunidades vegetales fue determinada de manera indirecta a través de cambios en la exposición de laderas y altitud, y donde se hallaron bosques de abeto en exposiciones desfavorables, aunque protegidos por una alta energía del relieve, como ocurre en cañadas y barrancos (García-Aguirre et al. 2010). Debido a esta causa se han identificado alteraciones en las propiedades del suelo y de la productividad de los sitios que inciden en las características de la vegetación (Newman et al. 2014). 
Es de interés, la existencia de un umbral dado por la energía del relieve, que coloca a los bosques de Carpinus tropicalis y Quercus delgadoana y los de C. tropicalis y Prunus serotina por debajo de los $66 \mathrm{~m}$, y al resto de las comunidades, por encima. Este valor también debe considerarse como un límite difuso ya que puede variar, aunque brinda un panorama general de la distribución de las comunidades mejor conservadas en las zonas intermedias del intervalo de distribución y fuera de los sectores más abruptos de la vertiente.

Estos resultados sugieren que las escalas regional y del paisaje son adecuadas para el estudio de la distribución de las comunidades del bosque (Tapia-Varela y López-Blanco 2002), incluso, de forma más clara y evidente que las variables relacionadas con la fragmentación, las cuales no tienen efectos significativos sobre la composición de las comunidades. Las variables de la fragmentación están altamente correlacionadas entre sí, lo cual es normal dadas las fórmulas para su obtención y su objetivo (McGarigal et al. 2012).

Este resultado es inesperado, ya que estudios previos demuestran que la fragmentación se relaciona con la disminución del número de especies a escala de paisaje, tanto por el aumento en el efecto de borde como por la disminución del tamaño de las “islas” (Hilty et al. 2006, Echeverría et al. 2007, Collinge 2009). De hecho, es innegable que la fragmentación genera una cierta heterogeneidad a escala local, la cual no tiene los efectos positivos que la heterogeneidad del relieve tiene sobre la biodiversidad (Slaymaker 2007), e incluso puede ser negativa al comprometer la continuidad del ecosistema y favorecer la inestabilidad ambiental y la vulnerabilidad biofísica (Hilty et al. 2006).

\section{CONCLUSIONES}

Se identifican dos comunidades del bosque húmedo de montaña (bosque de Carpinus tropicalis y Quercus delgadoana y bosque de Carpinus tropicalis y Prunus serotina), dos ecotonos en la parte baja y alta de la vertiente (ecotono con Alchornea latifolia y Liquidambar macrophylla y ecotono con Cupressus lusitanica y Alnus jorullensis, respectivamente), y un agrosistema dentro del ámbito del bosque (cafetales bajo dosel del bosque). La altitud y la energía del relieve tienen efectos significativos sobre la composición y riqueza de los sitios en estudio, y son dominantes sobre los factores de la fragmentación con los que se evaluaron. Esto puede indicar la presencia de niveles jerárquicos en los que actúan los factores del medio físico y es posible encontrar la escala a la que actúa cada factor tanto del relieve como de la fragmentación, sin embargo, son necesarios estudios posteriores, descartando los efectos del clima y el relieve para detectar los efectos a escalas más locales.

\section{AGRADECIMIENTOS}

Esta investigación fue financiada por el proyecto UNAM-DGAPA-PAPIIT IN301414.

\section{REFERENCIAS}

Alcántara O, I Luna, A Velázquez. 2002. Altitudinal distribution patterns of Mexican cloud forest based upon prefential characteristic genera. Plant Ecology 161: 167-174.

Cayuela L, D Golicher, J Rey-Benayas. 2006. The Extent, Distribution, and Fragmentation of Vanishing Montane Cloud Forest in the Highland of Chiapas, México. Biotropica 38(4): 544-554.

Challenger A, J Soberón. 2008. Los ecosistemas terrestres. In Capital Natural de México. Ciudad de México, México. Comisión Nacional para el Conocimiento y Uso de la Biodiversidad. p. 87-108.

Collinge S. 2009. Ecology of fragmented landscapes. Baltimore, USA. The JHU Press. p 340.

Corenblit D, A Gurnell, J Steiger, E Tabacchi. 2008. Reciprocal Adjustments Between Landforms and Living Organism: Extended Geomorphic Evolutionary Insights. Catena 73: 261-273.

Csorba P. 2010. Anthropogenic geomorphology and landscape ecology. In Szabo J, L David, D Loczy eds. Anthropogenic Geomorphology. Dordrecht, The Netherlands. Springer. p. 39-52.

Echeverría C, AC Newton, A Lara, JM Rey-Benayas, DA Coomes. 2007. Impacts of Forest Fragmentation on Species Composition and Forest Structure in the Temperate Landscape of Southern Chile. Global Ecology and Biogeography 16: 426-439.

Faith D, P Minchin, L Belbin. 1987. Compositional dissimilarity as a robust measure of ecological distance. Vegetatio 69: 57-68.

Farina A. 1998. Principles and Methods in Landscape Ecology. London, UK. Chapman \& Hall. p 411.

García E. 1973. Modificaciones al sistema de clasificación climática de Köppen. Segunda Edición. México D. F., México. UNAM - Instituto de Geografía. 246 p.

García-Aguirre M, R Álvarez, R Dirzo, MA Ortiz. 2010. Delineation of biogeomorphic land units across a tropical natural and humanized terrain in Los Tuxtlas, Veracruz, México. Geomorphology 121: 245-256.

García-García F, RA Montañez. 1991. Warm fog in eastern Mexico: a case study. Atmósfera 4: 53-64.

Hilty JA, WZ Lidicker Jr, AM Merenlender. 2006. Corridor ecology. The science and practice of linking landscapes for biodiversity conservation. Washington, USA. Island Press. p 323.

Hovenden MJ, T Brodribb. 2000. Altitude of origin influences stomatal conductance and therefore maximum assimilation rate in Southern Beech, Nothofagus cunninghamii. Functional Plant Biology 27(5): 451-456.

Huston M. 2005. The Three Phases of Land-Use Change: Implications for Biodiversity. Ecological Applications 15(6): 1864-1878.

Jules E, P Shahani. 2003. A broader ecological context to habitat fragmentation: why matrix habitat is more important than we thought. Journal of Vegetation Science 14: 459-464.

Kruskal JB. 1964. Nonmetric multidimensional scaling: a numerical method. Psychometrika 29(2): 115-129.

Ludwig J, J Reynolds. 1988. Statistical Ecology: a primer on methods and computing. New York, USA. Wiley-Interscience Publication. p 337.

McGarigal K, S Cushman, E Ene. 2012. FRAGSTATS v4: Saptial 
Pattern Analysis Program for Categorical and Continuous Maps. Consultado 05 de mayo 2015. Disponible en http:// www.umass.edu/landeco/research/fragstats/fragstats.html

Newman ME, KP McLaren, BS Wilson. 2014. Long-term socioeconomic and spatial pattern drivers of land cover change in a Caribbean tropical moist forest, the Cockpit Country, Jamaica. Agriculture, Ecosystems \& Environment 186: 185-200.

Rzedowski J. 2006. Vegetación de México. Consultado 17 dic. 2014. Disponible en http://www.conabio.gob.mx/institucion/centrodoc/doctos/vegetacion_de_mexico.html

SGM (Servicio Geológico Mexicano, MX). 2007. Carta Geológica-Minera Jalapa E14-B27, Veracruz. Pachuca, México. SGM. s.n.

Slaymaker O. 2007. The Potential Contribution of Geomorphology to Tropical Mountain Development: the Case of the MANRECUR Project. Geomorphology 87: 90-100.
Tapia-Varela G, J López-Blanco. 2002. Mapeo geomorfológico analítico de la porción central de la Cuenca de México: unidades morfogenéticas a escala 1:100,000. Revista Mexicana de Ciencias Geológicas 19: 50-65.

Toledo T. 2009. El Bosque de Niebla. Biodiversitas 83: 1-6.

Turner M, R Gardner R O’Neill. 2001. Landscape Ecology in Theory and Practice. New York, USA. Springer-Verlag. $482 \mathrm{p}$.

Williams-Linera G. 2007. El bosque de niebla del centro de Veracruz: ecología, historia y destino en tiempos de cambio climático. México DF, México. Instituto de Ecología, Comisión Nacional para el Conocimiento y Uso de la Biodiversidad. $204 \mathrm{p}$.

Wood S. 2003. Thin Plate Regression Splines. Journal of the Royal Statistical Society. Series B (Statistical Methodology) 65: 94-114.

Recibido: 19.08 .16

Aceptado: 08.09.16 
